\title{
Phase and Amplitude Contrast Microscopy in Partially Coherent Light
}

\author{
M. De and P. K. Mondal \\ Department of Applied Physics, University College of Technology, 92 Acharya Prafulla \\ Chandra Road, Calcutta 9, India
}

(March 10, 1965)

This paper describes the effects obtained in phase contrast imagery with partially coherent illumination of phase objects. The objects chosen for study include simple Zernike type of periodic phase gratings of the form (i) $A(u)=1+i \cdot \Delta \cdot \cos \omega u$ and (ii) $A(u)=1+i \cdot \sum_{n=1}^{\infty} \Delta_{n} \cdot \cos n \omega u$, with axial or oblique partially coherent illumination. The concept of "effective source" has been utilized, and the nonlinearity in the intensity transmission due to partial coherence has been analyzed in terms of "fundamental contrast" and "remnant."

\section{Introduction}

It is known that ordinary methods of observation are not suitable for obtaining information about phase objects. Special techniques have to be used to render phase variations visible, namely, the dark-ground method of observation in which the direct beam illuminating the object is stopped. There is the other method, the Schlieren method, which differs from the above in that not only the direct light but also a part of the diffracted beam is also stopped from entering the ultimate detector. However, the most powerful method in this respect is due to Zernike [1934, 1942] which makes use of a phase modulation of the central or zero order of the diffraction produced by the object. In fact, it was shown by Zernike that with this method, which he termed as "phase-contrast" method, phase variations in the object are practically linearly transformed into changes in intensity and thus made observable. Later, Hopkins [1952] pointed out that because of the finite size of the aperture, some details of phase structures could be seen in ordinary microscopes as well.

The Zernike theory of the phase contrast method basically makes use of the Abbé theory of microscopic imagery in coherent light. We shall then refer to this as the Abbé-Zernike theory. A simple and obvious extension may be made to the theory to include the effect of amplitude modulation of the diffraction spectra, thus including the dark-ground and Schlieren methods of observation as well. In the original elementary theory of Zernike there were certain implicit assumptions which were later treated by Picht [1936] and Kahn [1955]. Osterberg [1944] has also made a thorough study of the phase-contrast method as a problem in diffraction, in which he showed that the phase-plate characteristics could simply be treated as a "coating function" modifying the pupil function of the microscope objective. His basic diffraction integral, which he terms as "generalized transport integral" includes the effect of not fully coherent illumination, though in the final analysis he restricts himself to a narrow cone, or effectively coherent illumination of the object.

In the meantime, the concept of partial coherence has been utilized by Hopkins [1953] in formulating what he describes as the generalized Abbé theory, the generalization being due to his inclusion of the effect of partially coherent illumination of the object. Steel [1958] also gave a generalized treatment of image formation in partially coherent light. We followed up the obvious next step in generalizing the Abbé-Zernike theory of "phase and amplitude contrast microscopy in partially coherent light." As a first step in this direction De and Som [1963] extended the study of Hopkins [1952] to show that phase structures could be observed in ordinary microscopes even in partially coherent light, the extent of observation depending on the magnitude of phase variations and the degree of partial coherence. 
In this paper, we now discuss the effect of phase and amplitude contrast technique on the images of simple Zernike-type objects represented by $A(u)=1+i \Delta(u)$, in which the magnitude of $\Delta$ is small enough to justify the presumption that the object shows no variable intensity transmission. In our treatment, we have utilized the theorem of effective source as postulated by Hopkins [1953, 1957], and the observation of Osterberg that the phase and amplitude contrast plate characteristics may be simply associated with the pupil function of the objective. This treatment may be further extended to cover a general type of phase grating represented by $A(u)=\exp [i \Delta(u)]$, of which the Zernike-type object is just a special case. We may further assert that our method is perfectly amenable to the consideration of even nonperiodic objects in a rather straightforward manner, and also of the effects of small residual aberrations in the objectives. Slansky [1962] also studied a similar problem, though his treatment is restricted to the case of Zernike-type objects only.

\section{General Solution}

In deriving the general solution of the problems of diffraction in phase and amplitude contrast microscopy in partially coherent light, we shall make use of the formulation by Hopkins [1953]. In his so-called generalized Abbé theory in partially coherent light, Hopkins showed that, in all practical cases, the illumination of the object may be considered as due to a self-luminous source placed at the condenser exit pupil. This is termed the "effective source," and insofar as the degree of partial coherence on the object plane is concerned, there seems to be no difference of practical significance between the two types of illuminating systemsviz, the Kohler and the critical illumination. This and the further consideration of the progress of mutual intensity between a pair of points through the object to the objective aperture shows that this "effective source" may be orthoscopically projected on to the objective aperture through the object point.

Earlier Osterberg and Wilkins [1949] showed that with objectives satisfying Abbé's sine condition, the diffraction plate characteristics may be simply treated as modifying the pupil function of the objective. Thus, the overall pupil function of the phase- and amplitude-contrast objective may be taken as

$$
\bar{f}(x, y)=f(x, y) p(x, y)
$$

where $p(x, y)$ is the complex amplitude transmission of the diffraction plate. That this is possible is seen from the fact that $(x, y)$ denotes not only the coordinates of the point on the pupil but also the angles a ray makes with the axis as it comes from the object point on the axis to the point on the pupil. Thus, this also refers to a corresponding point on the back focal plane on which the diffraction plate is situated.

Based on these two premises, the final intensity distribution in the image plane is given by

$$
B^{\prime}\left(u^{\prime}, v^{\prime}\right)=\iint \gamma\left(x_{0}, y_{0}\right)\left|\phi\left(x_{0}, y_{0} ; u^{\prime}, v^{\prime}\right)\right|^{2} d x_{0} d y_{0}
$$

where

$$
\begin{gathered}
u^{\prime}=\frac{2 \pi}{\lambda} \cdot n^{\prime} \cdot \sin \alpha^{\prime} \cdot \xi^{\prime}, \\
v^{\prime}=\frac{2 \pi}{\lambda} \cdot n^{\prime} \cdot \sin \alpha^{\prime} \cdot \eta^{\prime}, \\
x_{0}=\frac{a_{0}}{h}, y_{0}=\frac{b_{0}}{h},
\end{gathered}
$$

and where

$u^{\prime} v^{\prime}$-reduced coordinates of a point on the image plane,

$\xi^{\prime}, \eta^{\prime}$-coordinates of a point on the image plane, 
$x_{0}, y_{0}$-fractional coordinates of a point on the pupil,

$\sin \alpha^{\prime}$-semiangular aperture of the objective referred to the object point,

$a_{0}, b_{0}$-coordinates of a point on the pupil or on the effective source,

$h$-radius of the pupil,

$n^{\prime}$-refractive index of the medium between the object and the objective,

$\gamma\left(x_{0}, y_{0}\right)$-is the effective source function described in terms of the pupil coordinates, $\left|\phi\left(x_{0}, y_{0} ; u^{\prime}, v^{\prime}\right)\right|^{2}$ - partial intensity at the point $\left(u^{\prime}, v^{\prime}\right)$ of the image plane due to an element $d x_{0} d y_{0}$ of the effective source.

The physical significance of the above integral is obvious. To obtain the partial intensity, $|\phi|^{2}$, we may proceed as follows:

Let $A(u, v)=$ complex amplitude transmission of the object, which may be Fourier analyzed into

$$
a(x, y)=\iint_{-\infty}^{+\infty} A(u, v) e^{-i(u x+v y)} d u d v
$$

giving the components of the spectrum of $A(u, v)$.

If we consider the object to be illuminated by an element $d x_{0} d y_{0}$ of the effective source at $x_{0}, y_{0}$, the spectrum would be shifted by a corresponding amount. Thus, the amplitude distribution of the spectrum would be given by $a\left(x-x_{0}, y-y_{0}\right)$, which, after passing through the objective and the diffraction plate, will be modified to $a\left(x-x_{0}, y-y_{0}\right) \vec{f}(x, y)$. The partial intensity, then, is given by the squared modulus of the inverse transform of the above. In other words,

$$
\phi\left(x_{0}, y_{0} ; u^{\prime}, v^{\prime}\right)=\iint a\left(x-x_{0}, y-y_{0}\right) \bar{f}(x, y) e^{i\left(u^{\prime} x+v^{\prime} y\right)} d x d y
$$

gives the complex amplitude distribution in the image plane due to an element of the source at $x_{0}, y_{0}$. It may also be interpreted as the complex amplitude distribution in the image plane when the object is illuminated by a plane wave front proceeding in the direction making an angle $x_{0}, y_{0}$ with the axis.

It now remains to evaluate explicitly the function $\phi$ pertaining to a specific object, which is possible if the composite pupil function is known.

\section{Solutions of Cases of Periodic Grating Objects of the Zernike Type}

We are now in a position to obtain solutions of specific cases. First, we shall consider those types of diffraction (or Zernike) plates which have a phase and amplitude contrast coating over an area which has the same relative size as the effective source. The complex amplitude transmission of this area, which we shall call the "conjugate area," is, say, given by $h e^{i \psi}$, while that of the remaining area, the so-called "complementary area," is unity. $h$ in the above implies amplitude contrast and $\psi$, phase contrast.

Let us first consider the simple Zernike-type object:

$$
A(u)=1+i \cdot \Delta(u)
$$

in which $\Delta$ is so small that $\Delta^{2}$ is neglected. In other words, the intensity transmission of the above object is constant while the phase varies as $\Delta(u)$. Assuming $\Delta(u)$ to be periodic, we have

where

$$
\Delta(u)=\sum \Delta_{n} \cdot \exp [-i n \omega u]
$$

$$
\Delta_{n}=\frac{1}{2 u_{0}} \int_{-u_{0}}^{+u_{0}} \Delta(u) \exp [i n \omega n] d u
$$

$2 u_{0}$ is the length of the fundamental period. $\omega=\left(\pi / u_{0}\right)$. 
The Fourier-spectrum of the object function (4) is thus given by

$$
a(x, y)=\delta(x, y)+i \sum_{-\infty}^{+\infty} \Delta_{n} \cdot \delta(x+n \omega, y)
$$

where $\delta(x, y)$ is the Dirac delta function.

Thus, we have for $\phi$ as defined in eq (3)

$$
\begin{aligned}
\phi\left(x_{0}, y_{0} ; u^{\prime}, v^{\prime}\right) & =\iint\left[\delta\left(x-x_{0}, y-y_{0}\right)+i \sum_{n=-\infty}^{+\infty} \Delta_{n} \cdot \delta\left(x-x_{0}+n \omega, y-y_{0}\right)\right] \bar{f}(x, y) \exp \left\{i\left(u^{\prime} x+v^{\prime} y\right)\right\} d x d y \\
& =\left[\bar{f}\left(x_{0}, y_{0}\right)+i \sum \Delta_{n} \cdot \bar{f}\left(x_{0}-n \omega, y_{0}\right) \exp \left\{-i n \omega u^{\prime}\right\}\right] \exp \left\{i\left(u^{\prime} x_{0}+v^{\prime} y_{0}\right)\right\}
\end{aligned}
$$

or

$$
|\phi|^{2}=\left|\bar{f}\left(x_{0}, y_{0}\right)\right|^{2}+2 \cdot \operatorname{Im}\left\{\bar{f}\left(x_{0}, y_{0}\right) \sum \Delta_{n}^{*} \bar{f}^{*}\left(x_{0}-n \omega, y_{0}\right) \exp \left(i n \omega u^{\prime}\right)\right\}
$$

in which the asterisk denotes the complex conjugate, and $\operatorname{Im}\{\ldots \ldots \ldots\}$ indicates that the imaginary part is to be taken. Terms involving $\Delta^{2}$ have been neglected. The final intensity would then be obtained by an integration of $|\phi|^{2}$ over the effective source $\gamma\left(x_{0}, y_{0}\right)$ as indicated in eq (1) above. The integration involved is of the form

$$
C\left(0, n_{0}\right)=\iint \gamma\left(x_{0}, y_{0}\right) \bar{f}\left(x_{0}, y_{0}\right) \bar{f}^{*}\left(x_{0}-n \omega, y_{0}\right) d x_{0} d y_{0}
$$

which is the generalized partially coherent response as described by Hopkins.

Since we shall be concerned with pupils or Zernike plates which are symmetrical about the $y_{0}$-axis, $\bar{f}\left(x_{0}+u \omega, y_{0}\right)=\bar{f}\left(x_{0}-u \omega, y_{0}\right)$ and if, further, we restrict ourselves to cases where $\Delta_{n}$ is real, then eq (7) reduces to the following simple form:

$$
|\phi|^{2}=\left|f\left(x_{0}, y_{0}\right)\right|^{2}+2 \sum_{u=1}^{\infty} \operatorname{Im}\left\{\bar{f}\left(x_{0}, y_{0}\right) \bar{f}^{*}\left(x_{0}-n \omega, y_{0}\right)\left(2 \Delta_{n}\right) \cos n \omega u^{\prime} .\right.
$$

The final intensity is thus given by

$$
B^{\prime}\left(u^{\prime}\right)=1+2 \sum_{n=1}^{N}\left(2 \Delta_{n}\right) \frac{\operatorname{Im}\{C(0, n)\}}{C(0,0)} \cos n \omega u^{\prime}
$$

in which a normalization is effected by the constant term $C(0,0)$. The $C$ 's in the above equation are as defined in eq (8). The summation has been shown to extend from 1 to $N$, where $N \omega \leq 1+\rho$ since $C(0, n)$ shall be 0 for larger values of $n$.

The problem now reduces simply to an evaluation of the $C$ 's with given Zernike plate and effective source characteristics.

\section{Evaluation of $C(0, n)$}

In this section we shall describe the mode of evaluation of $C(0, n)$. We shall consider first axial illumination of the object with a circular disk-shaped effective source. The radius of this source projected on the aperture of the objective is, say, $\rho$. The actual radius of the condenser aperture may be obtained from the following [viz: Som, 1963]:

$$
n_{c} \cdot \sin \alpha_{c}=n_{0} \cdot \sin \alpha_{0}=n_{0} \cdot \rho \cdot \sin \alpha
$$

where $n_{C}$ and $n_{0}$ are the refractive indices of the media between the condenser and the object, and between the object and the objective respectively, $\alpha_{C}$ is the semiangular aperture of the condenser from the object point, $\alpha_{0}$ is the corresponding semiangular aperture of the effective source projected on the objective, and $\alpha$ is the semiangular aperture of the objective. In what follows we shall consider only cases in which $\rho<1$. 
Second we shall consider annular illumination, i.e., cases of an annular condenser aperture with radii 1 and $\rho_{a}<1$. The objective shall be assumed to be free from aberrations. Further, the conjugate area of the Zernike plate shall have the same size and shape as that of the "effective source."

(i) Axial illumination:

Let us recall the expression for $C(0, n)$ in eq (8)

$$
C(0, n)=\iint \gamma\left(x_{0}, y_{0}\right) \bar{f}\left(x_{0}, y_{0}\right) \bar{f}^{*}\left(x_{0}-n \omega, y_{0}\right) d x_{0} d y_{0} .
$$

As indicated above, we may write

$$
\begin{aligned}
\gamma\left(x_{0}, y_{0}\right) & =1 \text { within a circle } \\
& =0 \text { outside. } \\
\bar{f}\left(x_{0}, y_{0}\right) & =f\left(x_{0}, y_{0}\right) p\left(x_{0}, y_{0}\right) \\
f\left(x_{0}, y_{0}\right) & =1 \text { within a circle } \\
& =0 \text { outside } \\
p\left(x_{0}, y_{0}\right) & =h e^{i \psi} \text { within a circle } \\
& =1 \text { outside. }
\end{aligned}
$$

Figure 1 shows the geometrical implication of the integral (8). Evidently the region of integration is complicated by the various limits imposed on the functions that appear in the integrand. The values of $C(0, n)$ have been obtained by subdividing the area of integration into different areas of overlap of two circles of same or different radii. These latter areas could be easily determined analytically, and with the values of the function within these different subdivided areas, one would obtain the final values of $C(0, n)$ in terms of $h, \psi, n, \omega, \rho$. They are given as follows:

$$
\begin{aligned}
\operatorname{Im}\{C(0, n)\} & =\left\{1-D_{0}\left(\frac{n \omega}{\rho}\right)\right\} h \sin \psi \cdot \pi \rho^{2} \quad \text { for } \omega \leq 1-\rho \\
& =\left\{1-D_{0}\left(\frac{n \omega}{\rho}\right)-\frac{1}{2} D_{0}\left(\frac{2 x_{2}}{\rho}\right)+\frac{1}{2 \rho^{2}} D_{0}\left(2 \cdot \overline{n \omega+x_{2}}\right)\right\} h \sin \psi \cdot \pi \rho^{2} \\
& =\left\{-D_{0}\left(\frac{n \omega}{\rho}\right)+\frac{1}{2} D_{0}\left(\frac{2 x_{2}}{\rho}\right)+\frac{1}{2 \rho^{2}} D_{0}\left(2 \cdot \overline{n \omega+x_{2}}\right)\right\} h \sin \psi \cdot \pi \rho^{2},
\end{aligned}
$$

where

$$
\begin{aligned}
x_{2} & =\frac{1-\rho^{2}-\pi^{2} \omega^{2}}{2 n \omega}, n \omega+x_{2}=\frac{\left(1-\rho^{2}\right)+n^{2} \omega^{2}}{2 n \omega} \quad \text { for } n \omega \geq 1-\rho^{2}, \\
C(0,0) & =\pi \rho^{2} \cdot h^{2}
\end{aligned}
$$

and

$$
D_{0}(x)=\frac{1}{\pi}\left[2 \cdot \cos ^{-1} \frac{x}{2}-\sin \cdot\left(2 \cos ^{-1} \frac{x}{2}\right)\right] .
$$

It should be noted that $D_{0}(x)$ gives the response of a perfect lens system imaging a selfluminous object. These values are already tabulated, and hence the formulas for $C(0, n)$ given as above permit a straightforward evaluation, and also may be analyzed for aberrational effects with relative convenience. 


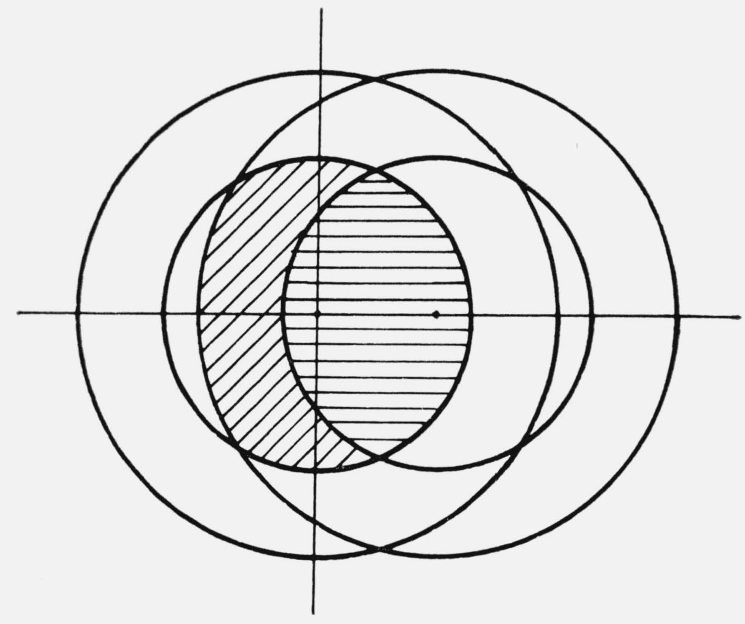

Figure 1. Evaluation of $\mathrm{C}(\mathrm{O}, \mathrm{n})$; axial illumination.

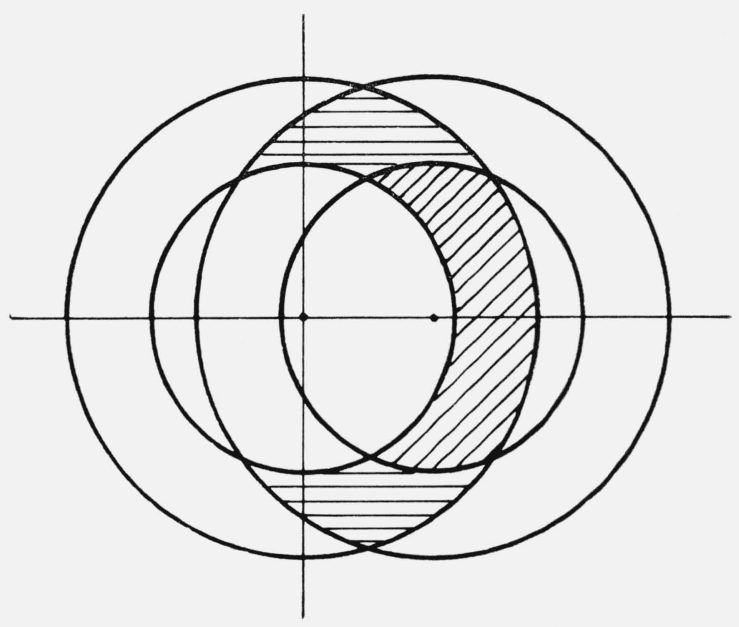

Figure 2. Evaluation of $\mathrm{C}(\mathrm{O}, \mathrm{n})$; annular illumination.

(ii) Annular illumination: In this case, we have

$$
\begin{aligned}
\gamma\left(x_{0}, y_{0}\right) & =1 \text { within an annulus bounded by circles } x_{0}^{2}+y_{0}^{2}=1 \text { and } x_{0}^{2}+y_{0}^{2}=\rho_{a}^{2} \\
& =0 \text { outside } \\
f\left(x_{0}, y_{0}\right) & =1 \text { within a circle } x_{0}^{2}+y_{0}^{2}=1 \\
& =0 \text { outside } \\
p\left(x_{0}, y_{0}\right) & =h e^{i \psi} \text { within an annulus bounded by circles } x_{0}^{2}+y_{0}^{2}=1 \text { and } x_{0}^{2}+y_{0}^{2}=\rho_{a}^{2} \\
& =1 \text { outside. }
\end{aligned}
$$

Figure 2 shows a typical geometrical representation of the area of integration in this case. The values of $C(0, n)$ are as follows:

$$
\begin{aligned}
\operatorname{Im}\{C(0, n)\} & =\pi \rho_{a}^{2}\left[1-D_{0}\left(\frac{n \omega}{\rho_{a}}\right)\right] h \sin \psi \quad \text { for } n \omega \leq 1-\rho_{a} \\
& =\pi \rho_{a}^{2}\left[1-D_{0}\left(\frac{n \omega}{\rho_{a}}\right)-\frac{1}{2} D_{0}\left(\frac{1-\rho_{a}^{2}-\pi^{2} \omega^{2}}{n \omega \rho_{a}}\right)+\frac{1}{2 \rho_{a}^{2}} D_{0}\left(\frac{1-\rho_{a}^{2}+n^{2} \omega^{2}}{n \omega}\right)\right] h \sin \psi \\
& =\pi \rho_{a}^{2}\left[-D_{0}\left(\frac{n \omega}{\rho_{a}}\right)+\frac{1}{2} D_{0}\left(\frac{1-\rho_{a}^{2}-n^{2} \omega^{2}}{n \omega \rho_{a}}\right)+\frac{1}{2 \rho_{a}^{2}} D_{0}\left(\frac{1-\rho_{a}^{2}+\pi^{2} \omega^{2}}{n \omega}\right)\right] h \sin \psi \\
& \text { for } 1-\rho_{a} \leq n \omega \leq \sqrt{1-\rho_{a}^{2}} \\
C(0,0)=\pi\left(1-\rho_{a}^{2}\right) h^{2} . & \text { for } n \omega \geq \sqrt{1-\rho_{a}^{2}} .
\end{aligned}
$$

(i) Axial illumination:

(a) Singly periodic grating:

We shall first consider the results for a singly periodic phase-grating. Such a grating is represented by

$$
\begin{aligned}
A(u) & =1+i \cdot \Delta \cdot \cos \omega u \\
& =1+i \cdot \Delta \cdot\left\{\frac{1}{2} \cdot e^{i \omega u}+\frac{1}{2} \cdot e^{-i \omega u}\right\} .
\end{aligned}
$$


Hence, from (9), we have

$$
B^{\prime}\left(u^{\prime}\right)=1+2 a_{1} \cdot \Delta \cdot \cos \omega u^{\prime}
$$

where

$$
a_{1}=\frac{\operatorname{Im}[C(0,1)]}{C(0,0)}
$$

With coherent illumination, i.e., in the limit of the effective source $\gamma\left(x_{0}, y_{0}\right)$ tends towards the Dirac delta function at the origin, $a_{1}$ tends towards $(\sin \psi / h)$ for all values of $\omega \leq 1$, so that the intensity distribution is given by

$$
B_{c}^{\prime}\left(u^{\prime}\right)=1+2 \cdot \frac{\sin \psi}{h} \cdot \Delta \cdot \cos \omega u^{\prime}
$$

Then the contrast in the image with partially coherent phase and amplitude contrast relative to that with a limitingly narrow-cone axial illumination is given by $a=\frac{a_{1} h}{\sin \psi}$. Further, with narrow-cone axial illumination, the contrast will be zero for $\omega \geq 1$, while in the partially coherent case, this limit is $\omega \geq 1+\rho$ when $a$ becomes zero. Thus, there is at least a possibility that there would be a gain in resolving power of the phase-contrast objective with a finite width of the illuminating beam. However, this is obtainable at the cost of contrast.

The values of $a$ have been calculated for various values of $\omega$ and $\rho$. Figure 3 shows the results graphically. The following summarizes some of the pertinent observations in respect of the above.

(1) So long as $2 \rho \leq \omega \leq 1-\rho$, the imagery in partially coherent light is indistinguishable from that with narrow-cone illumination in so far as the contrast is concerned. Hence, if one can have a prior knowledge of the range of frequencies in the object, it might be possible to choose

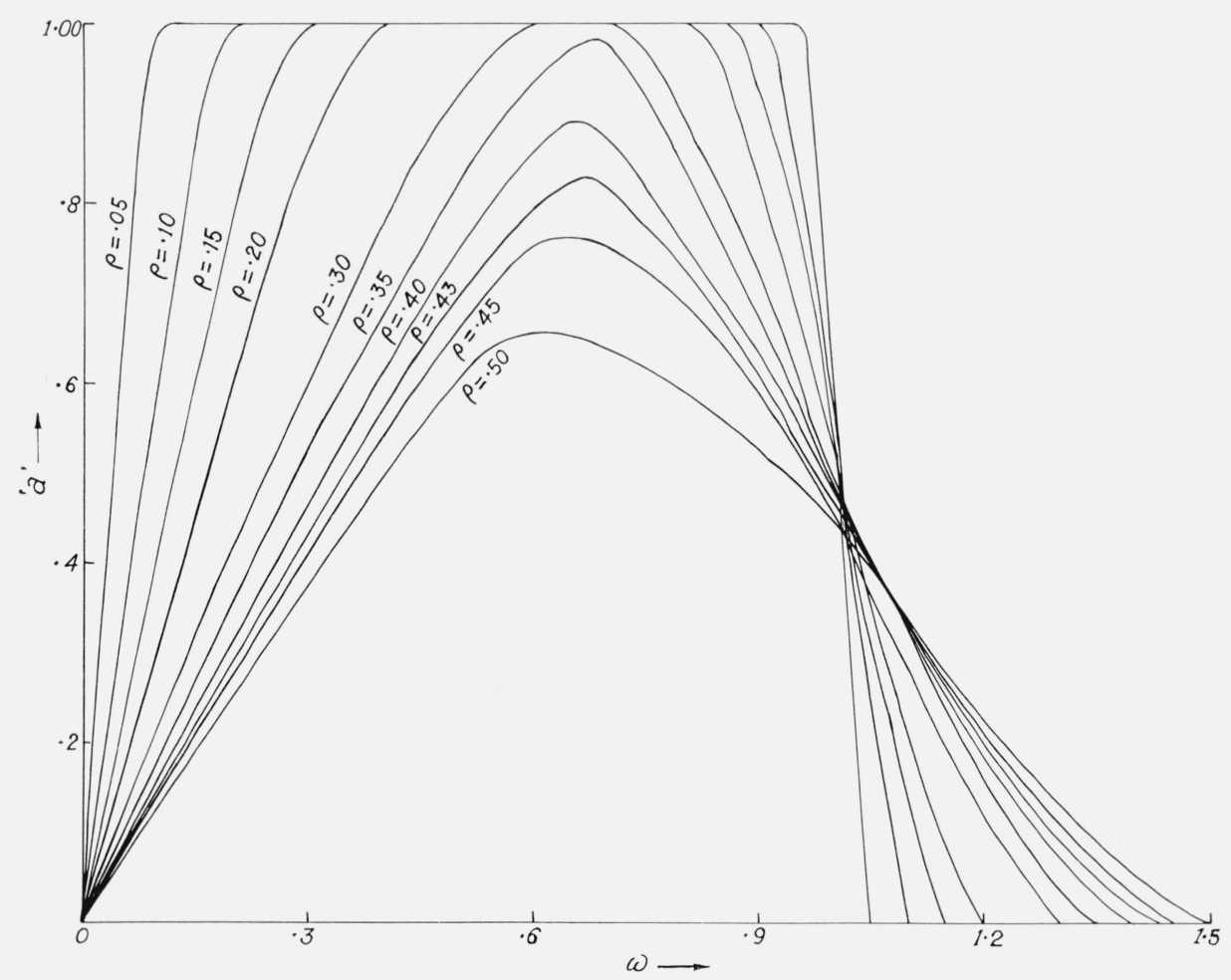

Figure 3. Contrast in the images of phase gratings; axial illumination. 
a finite size of $\rho$ to obtain a better photometric efficiency of the setup. Figure 4 a shows the formation of the spectra under these conditions. All the spectra are wholly within the pupil and completely separated. That is why the ultimate image shows no difference in contrast from what is obtainable with coherent illumination.

(2) If $\omega<2 \rho \leq 1-\rho$, the spectra are still wholly within the pupil, but they mutually overlap to a certain extent. Thus, not only the entire zero-order spectrum, but also parts of the first-order spectra are affected by the phase plate. This latter neutralizes the phase modulation, and hence contributes to a general loss in contrast. Figure $4 \mathrm{~b}$ represents the state of affairs under these conditions.

(3) If $1+\rho>\omega>1-\rho$, parts of the first-order spectra fall outside the pupil and hence do not contribute to the image formation. The result is a further decrease in contrast. Figure $4 \mathrm{c}$ represents these conditions.

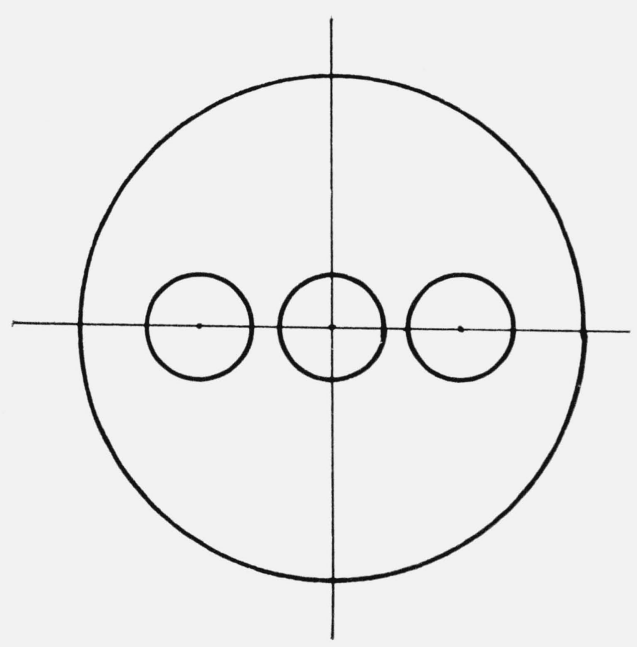

Figure 4a. Formation of the spectra: $2 \rho \leqslant \omega \leqslant 1-\rho$.

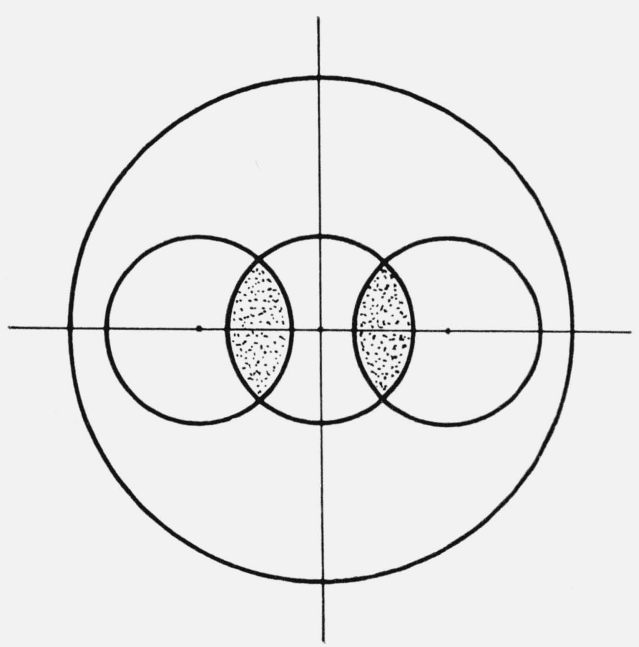

Figure 4b. Formation of the spectra; $\omega<2 \rho \leqslant 1-\rho$.

Figure 4c. Formation of the spectra; $1+\rho>\omega>1-\rho$. 
FIGURE 5. Useful frequency-range for partially coherent phase-contrast images.

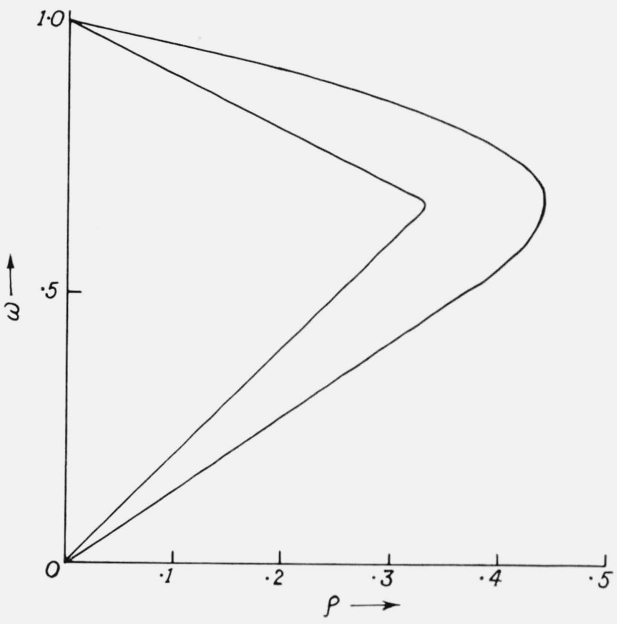

The values of $a$ as shown in figure 3 may now be analyzed in the light of the above observations. Figure 5 shows (i) the range of frequencies which shall have a contrast same as in the coherent case for different values of $\rho$, and (ii) the range of frequencies for which the relative contrast is equal to 0.8. This gives one an idea of the permissible maximum size of source that can be used for a satisfactory phase contrast image.

The curves in figures 3 and 5 have been drawn for cases when $h=1$, that is, when no amplitude modulation is used. Further $\sin \psi$ has been taken as unity as well. This implies that the phase of the zero-order spectrum is advanced or retarded by $\pi / 2$. Noting that $a_{1}$ is proportional to $\sin \psi$, the maximum phase contrast efficiency is obtained at this value. However, $a_{1}$ is seen to be inversely proportional to $h$, meaning thereby that the contrast may be enhanced by using a slight amplitude contrast as well. This, of course, is possible at the cost of the mean level of illumination of the final image.

(b) Square-topped phase-grating:

This is the case of a simple periodic structure where troughs and crests represent differences in optical path only.

Let

$$
A(u)=1+i \Delta(u)
$$

where $\Delta(u)$ is periodic in $u$ with a fundamental length of period $2 u_{0}$. Let, over one such fundamental interval,

$$
\begin{aligned}
\Delta(u) & =0 & & \alpha u_{0} \leq|u| \leq u_{0} \\
& =\Delta & & |u| \leq \alpha u_{0} .
\end{aligned}
$$

The Fourier spectrum is given by

$$
\Delta_{n}=\Delta_{-n}=\frac{\sin \alpha n \pi}{n \pi} \Delta .
$$

$\alpha$ in the above gives the ratio between the line width to line separations. Hence, from eq (9). we have

where $N \omega \leq 1+\rho$.

$$
B^{\prime}\left(u^{\prime}\right)=1+2 \Delta \cdot \sum_{n=1}^{N} 2 \cdot \frac{\sin \cdot \alpha \cdot n \pi}{n \pi} \cdot\left[\frac{\operatorname{Im}\{C(0, n)\}}{C(0,0)}\right] \cos n \omega u^{\prime}
$$


In the limit of a point source, the expression for intensity will be

$$
B^{\prime}\left(u^{\prime}\right)=1+2 \Delta \sum_{n=1}^{N}\left(2 \cdot \frac{\sin \cdot \alpha n \pi}{n \pi}\right)\left(\frac{\sin \psi}{h}\right) \cos \cdot n \omega u^{\prime}
$$

where $N \omega \leq 1$ since $\operatorname{Im}[C(0, n)]$ in the coherent limit will be $h \sin \psi$ for all values of $n \omega \leq 1$.

Figures 6 a to $d$ show the image of such gratings $(\Delta=0 \cdot 5)$ for four different frequencies, and for various effective source sizes at each frequency. The dotted curves represent an ideal case of linear unitary transformation of phase variation into amplitude variation.

The expression for $B^{\prime}\left(u^{\prime}\right)$ in eq $(19)$ or for $B_{c}^{\prime}\left(u^{\prime}\right)$ in eq $(20)$ shows that the final image is given by a truncated Fourier series, and hence one would expect to observe the so-called Gibbs' phenomenon of large oscillations in the region of the edges of the square-topped grating. In the presence of harmonics in the image intensity distributions it is indeed a bit difficult to define a unique contrast for such cases. The presence of these harmonics is in fact an indication of the effect of nonlinearity in the intensity transmission in partially coherent light, and as yet no unique specification has been suggested for a proper measure of such nonlinear effects. One suggestion is to express the contrast in terms of the fundamental frequency distribution, and lump together the effects of higher harmonics in a so-called "remnant" [Ingelstam, 1964]. This "remnant" may be described as the square root of the sum of the squares of all coefficients other than the constant and that of the fundamental frequency component. Figure 7 gives a plot of this fundamental contrast and the remnant against source sizes for different frequencies.

It is of interest to note that the remnant always decreases with increasing frequency, falling gradually down to zero. In fact, if $N<2$, there are no harmonics in the image intensity distribution. Thus, for a frequency up to 0.5, harmonic distortion would be present for all source sizes, though its magnitude as reflected in the value of the "remnant" may be negligibly small. For values of $\omega$ lying between 0.5 and 1 , the "remnant" would be zero only if $\rho$ is less than a certain value given by $\rho<2 \omega-1$.

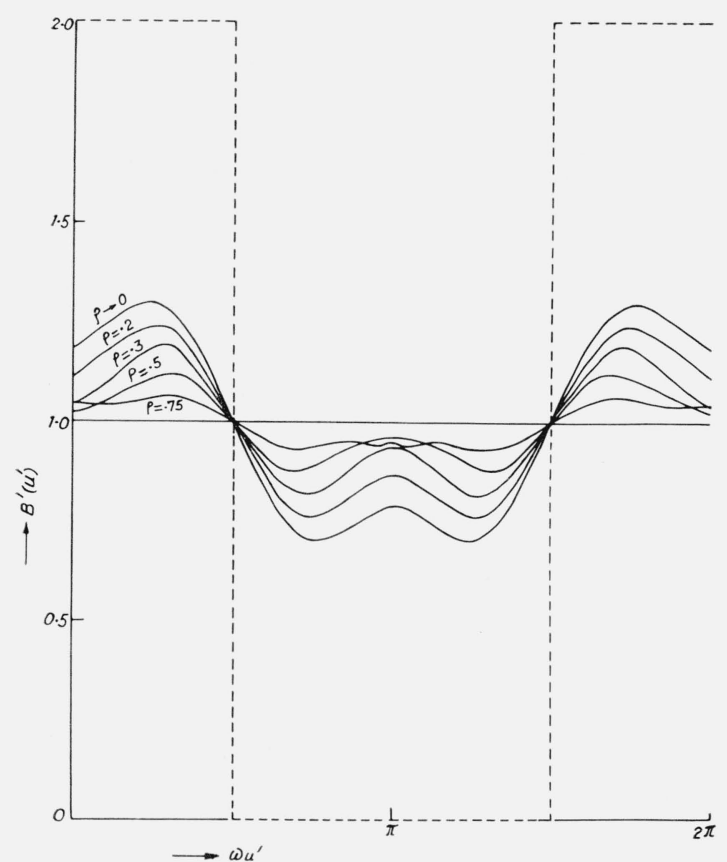

FIGURE 6a. Images of square-topped phase gratings; axial illumination; $\omega=0.25$.

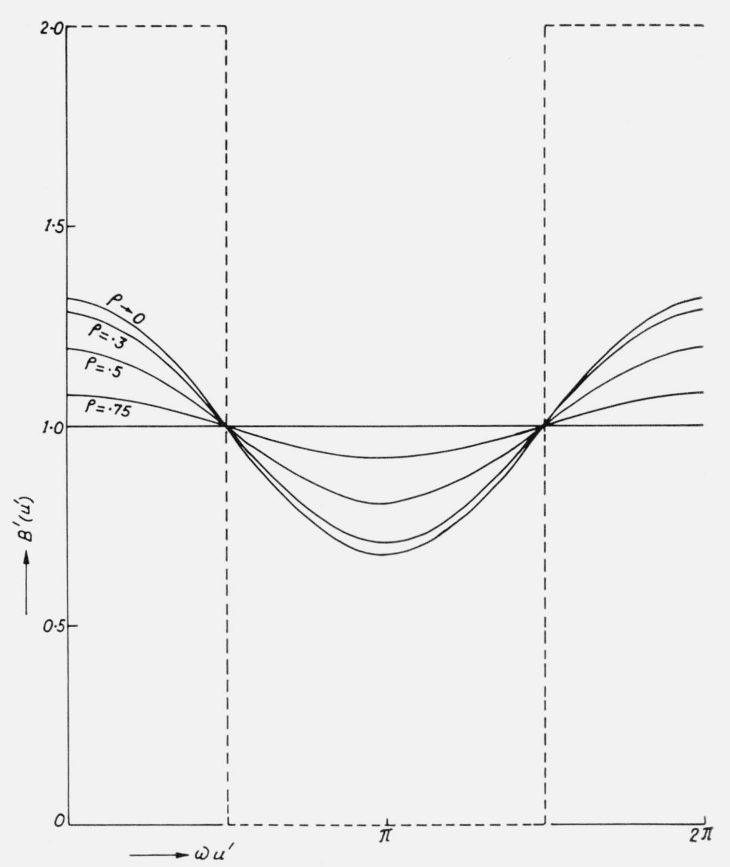

FIGURE 6b. Images of square-topped phase gratings; axial illumination; $\omega=0.50$. 


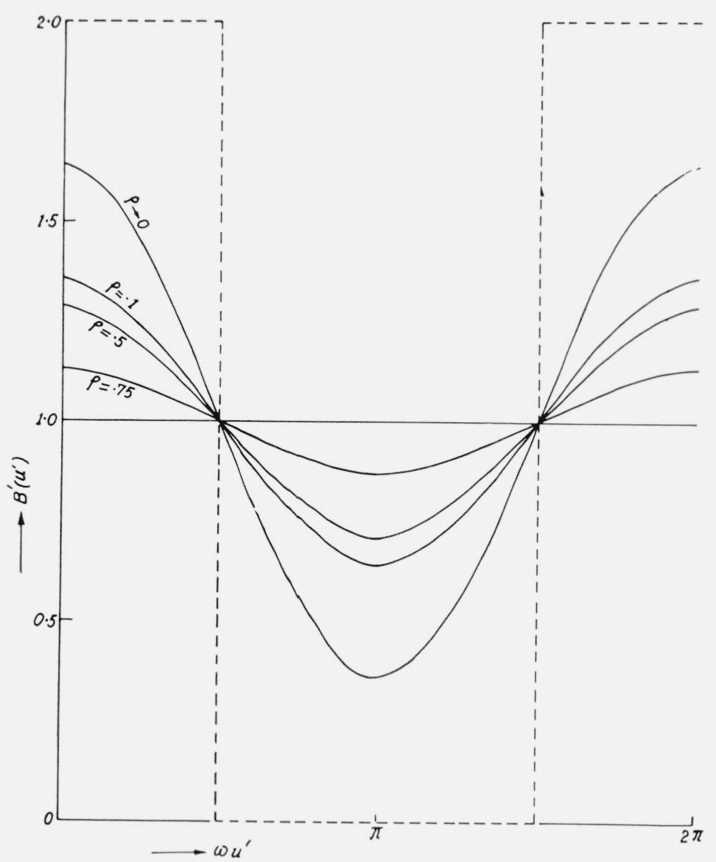

FIGURE 6c. Images of square-topped phase gratings; axial illumination; $\omega=1.0$.

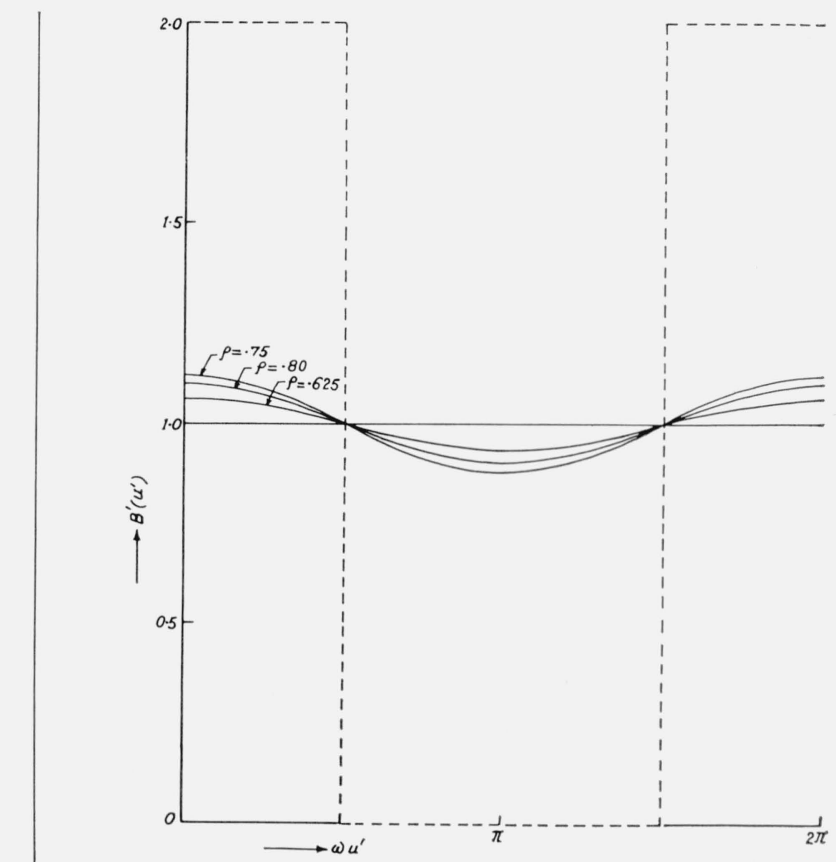

Figure 6d. Images of square-topped phase gratings;
axial illumination; $\omega=1.5$.

Figure 6d. Images of square-topped phase"gratings;
axial illumination; $\omega=1.5$.

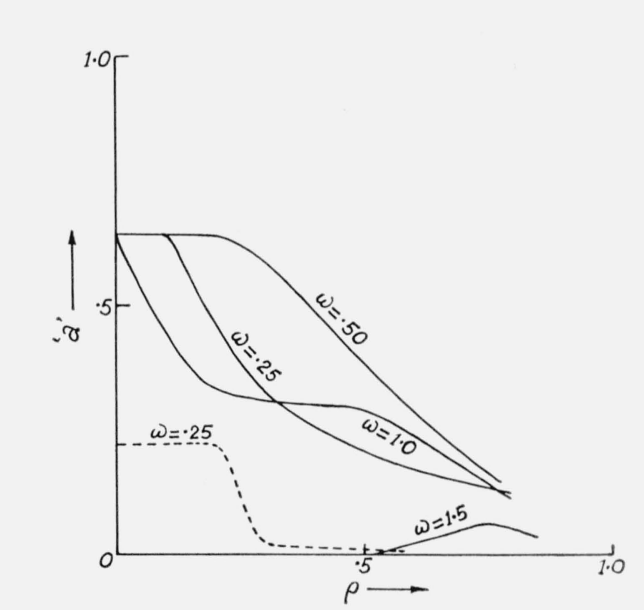

Figure 7. "Fundamental contrast", and "remnants" in the images of square-topped phase gratings; axial illumination.

In the cases for which computation has been done, $\alpha$ has been chosen as equal to $1 / 2$, implying equal widths for the troughs and crests of the phase grating. In these cases, the spectra consist of only the odd harmonic components and hence the "remnant" would be zero if $N<3$; i.e., if $\rho<3 \omega-1, \omega>0.33$. This expresses the range of validity of using the criterion of "fundamental contrast" for the study of the efficiency of phase-contrast devices in partially coherent light.

(ii) Annular illumination:

(a) Singly periodic grating.

The expression for intensity is the same as in the previous case, except that the values of $\operatorname{Im}[C(0, n)]$ to be used are as given in $(13)$. 
If the width of the annular source tends towards an infinitesimally small value, the area integral for $C(0, n)$ will tend towards a line integral, and we shall have

$$
\begin{aligned}
& \lim _{\rho_{a \rightarrow 1}} \operatorname{Im}[C(0, n)]=2 \cdot \cos ^{-1} \cdot\left(\frac{n \omega}{2}\right) h \sin \psi \\
& \lim _{\rho_{a \rightarrow 1}}[C(0,0)]=2 \pi h^{2} .
\end{aligned}
$$

Thus, unlike the case of axial illumination discussed above, we have in this case a contrast varying with frequency even with a source of infinitesimally narrow width; we have, in this limiting case,

$$
B_{c}^{\prime}=1+2 \Delta \cdot\left(\frac{\cos ^{-1} \cdot \frac{n \omega}{2}}{\pi}\right)\left(\frac{\sin \psi}{h}\right) \cos \omega u^{\prime} .
$$

The difference with the corresponding expression (16) for the axial case is to be noted with interest.

Evidently in this case the limiting resolvable frequency is $n \omega \leq 2$. This is just a theoretical limit, as the contrast also tends towards zero at the limit.

If we now consider the case of the annular source with a finite width, we shall find that Im $[C(0, n)]$ becomes zero for $n \omega>1+\rho_{a}$ which implies that we lose on the count of ultimate resolving power. Figure 8 shows graphically the values of $a_{n}=\operatorname{Im}[C(0, n)] / C(0,0)$ as a function of frequency $\omega$ for various values of $\rho_{a}$. The dashed curve in figure 8 indicates the range of frequencies which are so imaged that the relative contrast is nowhere less than 0.8 .

(b) Square-topped grating:

As in the case of axial illumination, we have, in the limit of a line source coinciding with the margin of the pupil,

where

$$
B_{c}^{\prime}\left(u^{\prime}\right)=1+2 \Delta \sum_{n=1}^{N}\left(\frac{\sin \cdot \alpha \cdot n \pi}{n_{0} \pi}\right)\left(\frac{2 \beta_{n}}{\pi}\right)\left(\frac{\sin \psi}{h}\right) \cos \cdot n_{\omega} \omega u^{\prime}
$$

$$
\beta_{n}=\cos ^{-1} \cdot \frac{n \omega}{2} \text { and } N \leq\left(1+\rho_{a}\right) / \omega
$$

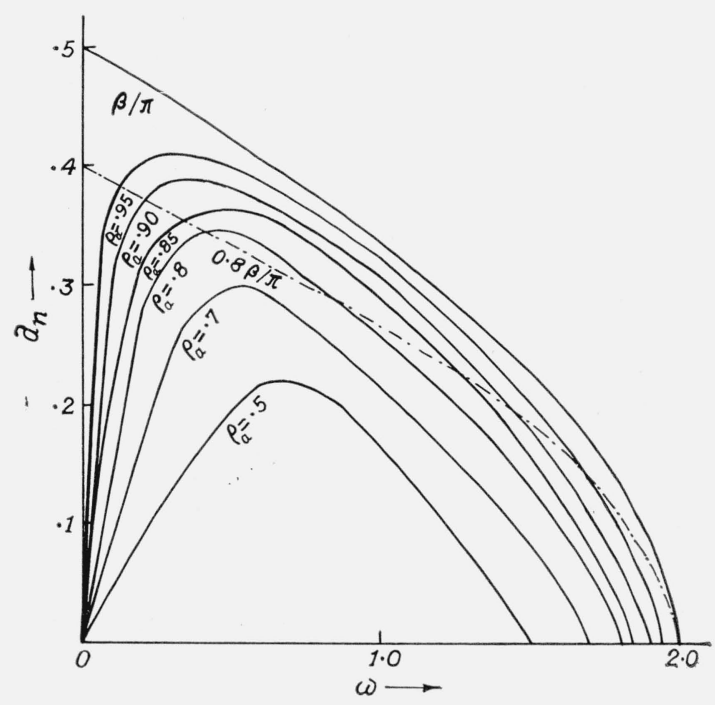

Figure 8. Contrast in the images of phase gratings; annular illumination. 
With a finite width of the source, we have an expression similar to (20) above. Figures 9a to d show the images of such gratings $(\Delta=0.5)$ of a number of frequencies $\omega=\frac{\pi}{u_{0}}$, where $2 u_{0}$ is the length of the period, and $\alpha=\frac{1}{2}$ implies equal widths for the troughs and crests of path-differences

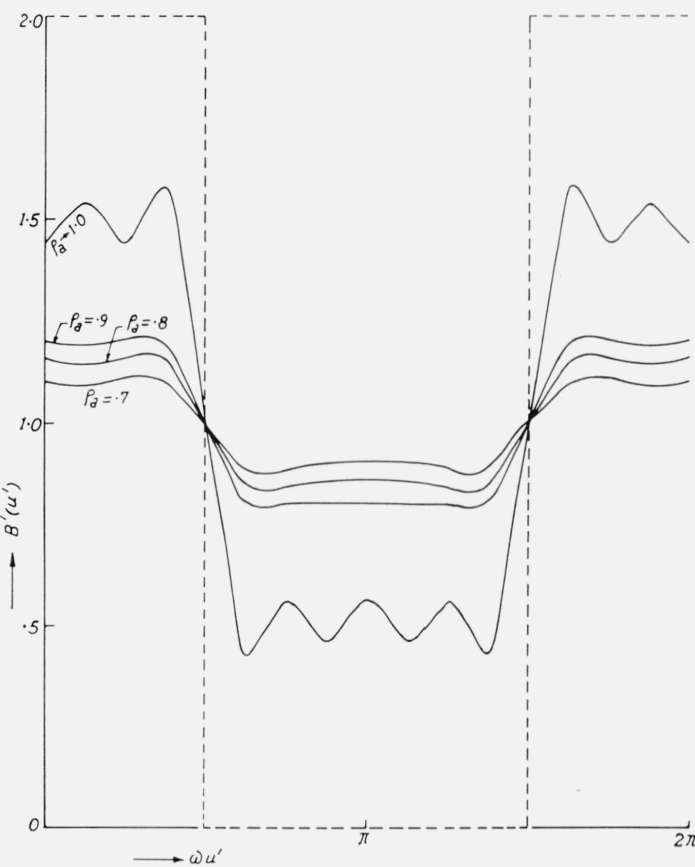

Figure 9a. Images of square-topped phase gratings; annular illumination; $\omega=0.25$.

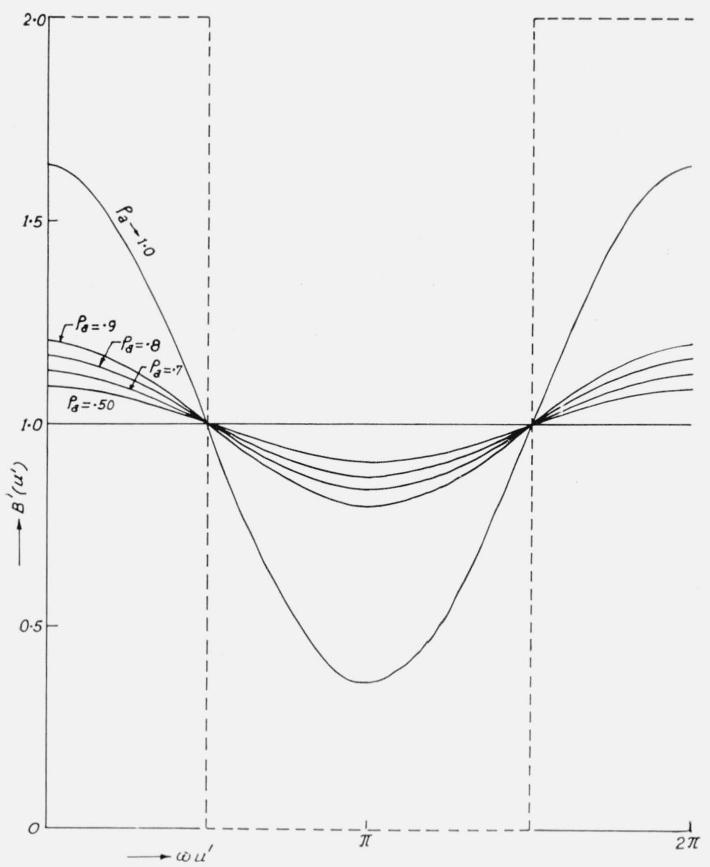

Figure 9c. Images of square-topped phase gratings; annular illumination; $\omega=1.0$.

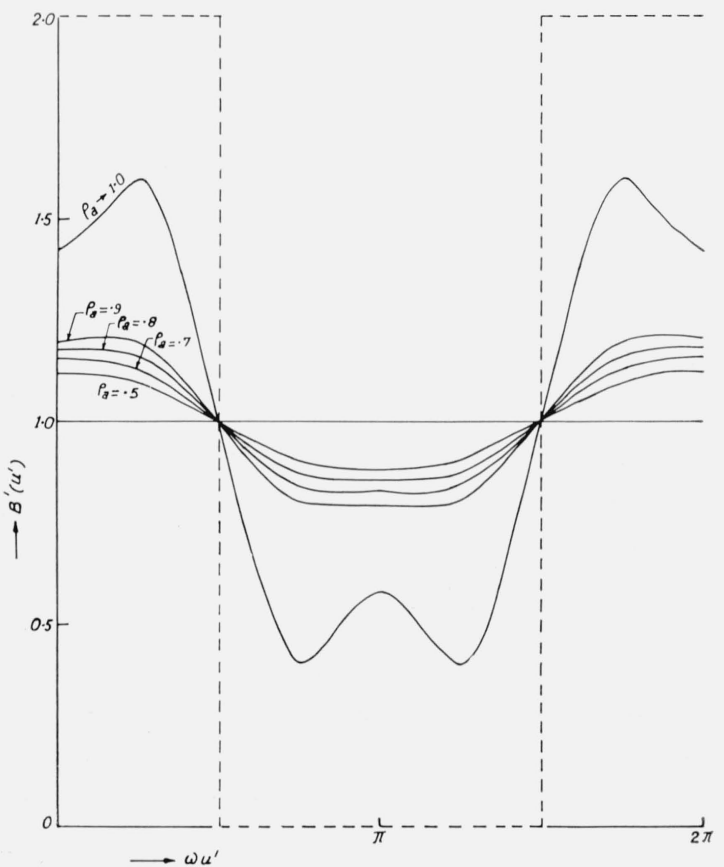

Figure 9b. Images of square-topped phase gratings; annular illumination; $\omega=0.50$.

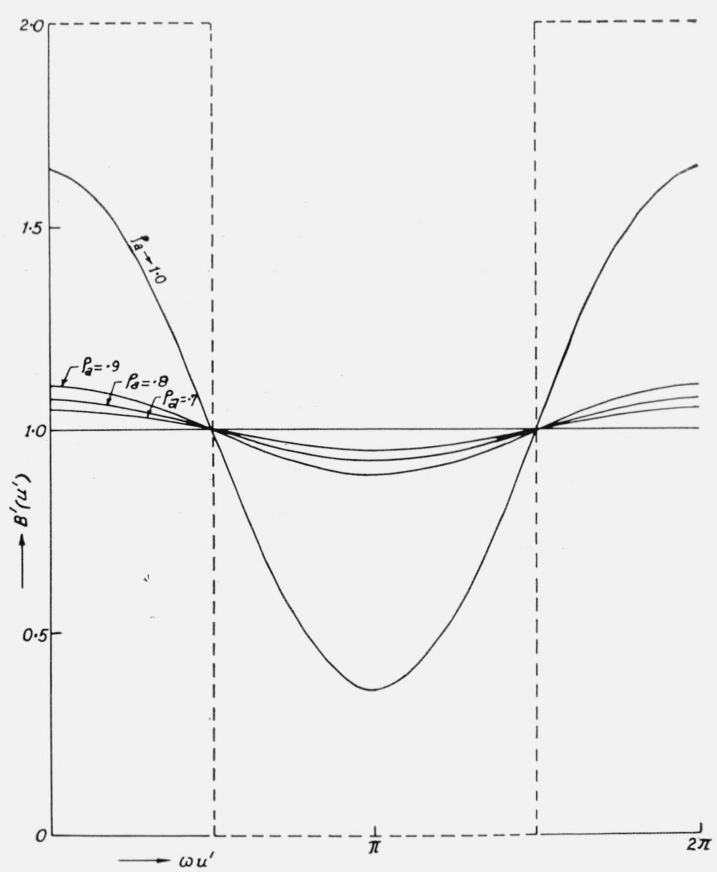

FIGURE 9d. Images of square-topped phase gratings; annular illumination; $\omega=1.5$. 


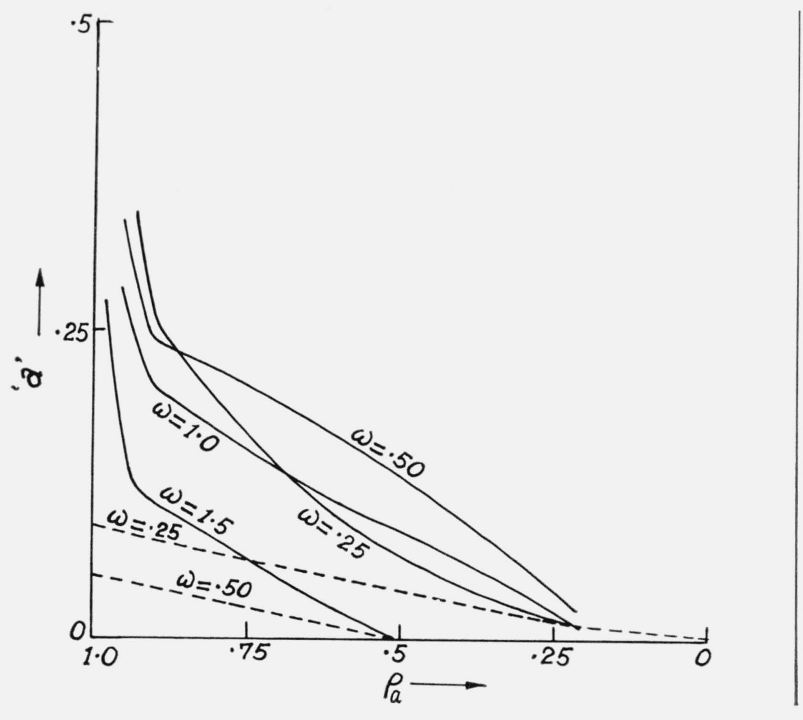

FIGURE 10. "Fundamental contrast" and "remnants" in the images of square-topped phase gratings; annular illumination.

in the grating. The dotted curves represent an ideal case of linear unitary transformation of phase variation into amplitude variation. Here also we may make the same remarks as done in the case of axial illumination of square-topped gratings. Figure 10 describes the situation, and the conclusions are also similar. Remembering that the coherent limit of resolution in this case is 2 , we find that practically the upper half of the range of resolvable frequencies is still assessable in terms of the "fundamental contrast" only.

This study has been undertaken under the sponsorship of the National Bureau of Standards. The authors are deeply indebted to the stimulating discussion with S. C. Som on the various aspects of this study.

\section{References}

De, M. and Som, S.C. (1963), J. Opt. Soc. Am. 53, 779.

Hopkins, H.H. (1952), Rev. Opt. (Paris), 142.

Hopkins, H.H. (1953), Proc. Roy. Soc. A217, 408.

Hopkins, H.H. (1957), J. Opt. Soc. Am. 47, 508.

Ingelstam, E., "Attempts to treat non-linear imaging devices" an abridged version of an invited paper given at the ICO Conference on Photographic and Spectroscopic Optics in Tokyo and Kyoto, September, 1964.

Kahn, F.D. (1955), Proc. Phys. Soc. B 68, 1073.

Osterberg, H. (1944), J. Opt. Soc. Am. 34, 773.

Osterberg, H. and Wilkins, J. (1949), J. Opt. Soc. Am. 39, 553.

Picht, J. (1936), Z. Instrumentenk. 56, 481.

Slansky, S. (1962), Opt. Acta. 9, 277.

Steel, W.H. (1958), Proc. Roy. Soc. A249, 574.

Zernike, F. (1934), Physica 1, 689.

Zernike, F. (1942), Physica 9, 686, 974.

(Paper 69C3-200) 